\title{
Protein Folding as a Physical Stochastic Process*
}

\author{
Kerson Huang \\ Physics Department, Massachusetts Institute of Technology \\ Cambridge, MA, USA 02139
}

June 27, 2021

\begin{abstract}
We model protein folding as a physical stochastic process as follows. The unfolded protein chain is treated as a random coil described by SAW (self-avoiding walk). Folding is induced by hydrophobic forces and other interactions, such as hydrogen bonding, which can be taken into account by imposing conditions on SAW. The resulting model is termed CSAW (conditioned self-avoiding walk. Conceptually, the mathematical basis is a generalized Langevin equation. In practice, the model is implemented on a computer by combining SAW and Monte Carlo. To illustrate the flexibility and capabilities of the model, we consider a number of examples, including folding pathways, elastic properties, helix formation, and collective modes.
\end{abstract}

\section{Introduction}

One of the outstanding unsolved problems in molecular biology is protein folding [1] 2. The principle through which the amino acid sequence determines the native structure, as wells as the dynamics of the process, remain open questions. Generally speaking, there have been two types of approaches to the problem: bioinformatics [3] and molecular dynamics (MD) 4 .

Bioinformatics is purely data analysis, and does not involve dynamics at all. It massages the data base of known proteins in different ways, using very sophisticated computer programs, in order to discover correlations between sequence and structure. By its very nature, it cannot provide any physical understanding.

On the other hand, MD solves the Newtonian equations of motion of all the atoms in the protein on a computer, using appropriate inter-atomic potentials. To describe the solvent, one inculdes thousands of water molecules explicitly, treating all the atoms in the water on same footing as those on the protein chain. Not surprisingly, such an extravagant use of computing power is so inefficient that one can follow the folding process only to about a microsecond, whereas the folding of a real protein takes from one second to ten minutes.

*Invited talk at BIOMAT 2007 International Symposium on Mathematical and Computational Biology, Zfmzcao dos Buzios, RJ, Brazil, 24th-29th Nov, 2007 
We shall try an approach from the point of view of statistical mechanics $[5]$. After all, the protein is a chain molecule immersed in water, and, like all physical systems, will tend towards thermodynamic equilibrium with the environment. Our goal is to design a model that embodies physical principles, and at the same time amenable to computer simulation in reasonable time.

We treat the protein as a chain performing Brownian motion in water, regarded as a medium exerting random forces on the chain, with the concomitant energy dissipation. In addition, we include regular (non-random) interactions within the chain, as well as between the chain and the medium.

The unfolded chain is assumed to be a random coil described by SAW (selfavoiding walk), as suggested by Flory [6] some time ago. That is, each link in the chain corresponds to successive random walks, in which the chain is prohibited from revisiting an occupied position. Two types of interactions are included in our initial formulation:

- the hydrophobic action due to the medium, which causes the chain to fold;

- the hydrogen-bonding within the chain, which leads to helical structure.

Other interactions can be added later.

We model the protein chain in 3D space, keeping only degrees of freedom relevant to folding, which we take to be the torsional angles between successive links. In the computer simulation, we first generate an ensemble of SAW's, and then choose a subensemble through a Monte Carlo method, which generates a canonical ensemble with respect to a Hamiltonian that specifies the interactions. We call the model CSAW [7] [8] (conditioned self-avoiding walk). Mathematically speaking, it is based on a Langevin equation [5] describing the Brownian motion of a chain with interaction. There seems little doubt that such an equation does describe a protein molecule in water, for It is just Newton's equation with the environment treated as a stochastic medium.. The model can be implemented efficiently on a computer, and is flexible enough to be used as a theoretical laboratory.

Both CSAW and MD are based on Newtonian mechanics, and differ only in the idealization of the system. In CSAW we replace the thousands of water molecules used in MD by a stochastic medium - the heat reservoir of statistical mechanics. We ignore inessential degrees of freedom, such as small fluctuations in the lengths and angles of the chemical bonds that link the protein chain. The advantages of these idealizations are that

- we avoid squandering computer power on irrelevant calculations;

- we gain a better physical understanding of the folding process.

One often hears a debate on whether the folding process is "thermodynamic" or "kinetic". There is also an oft cited "Levinthal paradox", to the effect that the folding time should be much larger than the age of the universe, since the protein (presumably) had to search through an astronomically large number of 
states before finding the right one. From our point of view, these are not real issues.

The question of thermal equilibrium merely hangs on whether the protein can reach equilibrium in realistic time, instead being trapped in some intermediate state. For any particular protein, simulation of the Langevin equation will answer the question.

As to Levinthal's "paradox", the protein is blithely unaware of that. It just follows pathways guided by the Langevin equation.

After a brief review of the basics of protein folding and stochastic processes, we shall describe the model in more detail, and illustrate its use through examples involving realistic protein fragments. We will demonstrate folding pathways, elastic properties, helix formation, and protein collective modes.

The results indicate that the model has been successful in describing qualitative features of folding in simple proteins.

\section{Protein basics}

\subsection{The protein chain}

The protein chain consists of a sequence of units or "residues", which are amino acids chosen from a pool of 20 . This sequence is called the primary structure. The center of each amino acid is a carbon atom called $C_{\alpha}$. Along the protein chain, the $C_{\alpha}$ 's are connected by covalent chemical bonds in the shape of a "crank" that lies in one plane. Two cranks join at a $C_{\alpha}$ with a fixed angle between them, the tetrahedral angle $\theta_{\text {tet }}=-\arccos (1 / 3) \approx 110^{\circ}$. The amino acids differ from each other only in the side chains connect to the $C_{\alpha}$ 's. There are 20 possible choices for side chains.

The relative orientation of successive cranks is determined by two torsional angles $\phi$ and $\psi$, as schematically illustrated in Fig.1. These torsional angles are the only degrees of freedom relevant to protein folding, and small oscillations in bond lengths and bond angles can be ignored. For our purpose, therefore, a protein of $N$ residues has $2(N-1)$ degrees of freedom.

\subsection{Secondary and tertiary structures}

At high temperatures, or in an acidic solution, the protein exists in an unfolded state that can be represented by a random coil [6]. When the temperature is lowered, or when the solution becomes aqueous, it folds into a "native state" of definite shape. Fig.2 shows the native state of myoglobin with different levels of detail. Local structures, such as helices, are called secondary structures. When these are blurred over, one sees a skeleton called the tertiary structure.

Secondary structures are of two main types, the alpha helix and the beta sheet, as shown in Fig.3. The former is stabilized by hydrogens bonds that connect residues 1 to 4,2 to 5 , etc. The beta sheet is a global mat sewn together by hydrogen bonds. 
Figure 1: Schematic representation of the protein chain. Centers of residues are carbon atoms labeled $\alpha$. They are connected by rigid chemical bonds in the shape of a planar crank. The only degrees of freedom we consider are the torsional angles $\phi, \psi$ that specify the relative orientations of successive cranks. Residues can differ only in the side chains labeled $R_{i}$, chosen from a pool of twenty. Atoms connected to the cranks are omitted for clarity.

Figure 2: Native state of Myoglobin showing different degrees of detail. 
Figure 3: Secondary structures. Dotted lines in the alpha helix denote hydrogen bonds. The beta sheet is composed of "beta strands" matted together by hydrogen bonds. two ajacent strands are connect by a "beta hairpin".

\section{$2.3 \quad$ Hydrophobic effect}

The molecules of liquid water form hydrogen bonds with each other, resulting in a dense fluctuating network, in which bonding partners change on a time scale of $10^{-12}$ s. A computer simulation of such a network is shown in Fig.4a 12. A foreign molecule introduced into water disrupts the network, unless it can participate in hydrogen bonding. If it can hydrogen-bond with water, it is said to be "soluble", or "hydrophilic", and will be received by water molecules as one of their kind. Otherwise it is unwelcome, and said to be "insoluble", or "hydrophobic". Protein side chains can be hydrophilic or hydrophobic.

When immerse in water, the protein chain folds in order to shield the hydrophobic residues from water. In effect, the water network squeezes the protein into shape. This is called the "hydrophobic effect". However, a "frustration" arises in this process, because the skeleton is hydrophilic, and likes to be in contact with water, as indicated in Fig.4b. The frustration is resolved by the formation of secondary structures, which use up hydrogen bonds internally. The folded chain reverts to a random coil when the temperature becomes too high, or when the $\mathrm{pH}$ of the solution becomes acidic.

\subsection{Folding stages}

As depicted schematically in Fig.5, a typical folding process consists of a very rapid collapse into an intermediate state called the "molten globule". The latter takes a relatively long time to undergo fine adjustments to reach the native state. The collapse time is generally less than $200 \mu \mathrm{s}$, while the molten globule can 
Figure 4: (a) Computer simulation of network of hydrogen bonds in liquid water. (b) The hydrophobic side chains $R_{1}$ and $R_{2}$ cannot form hydrogen bonds, and prefer to be shielded from water. However, the atoms $O$ and $H$ on the main chain need to form hydrogen bonds. A "frustration" thereby arises, and is resolved by formation of secondary structures that use up hydrogen bonds internally.

last as long as 10 minutes.

\subsection{Statistical nature of the folding process}

We have to distinguish between protein assembly inside a living cell (in vivo), and folding in a test tube (in vitro). In the former the process takes place within factory molecules called ribosomes, and need the assistance of "chaperon" molecules to prevent premature folding. In the latter, the molecules freely fold or unfold, reversibly, depending on the $\mathrm{pH}$ and the temperature.

We deal only with folding in vitro, in which ten of thousands of protein molecules undergo the folding process independently, and they do not fold in unison. We are thus dealing with an ensemble of protein molecules, in which definite fractions exist in various stages of folding at any given time. The Langevin equation naturally describes the time evolution of such an ensemble. Behavior of individual molecules fluctuate from the average, even after the ensemble has reached equilibrium. In macroscopic systems containing the order of $10^{23}$ atoms, such fluctuations are unobservably small. For a protein with no more than a few thousand atoms, however, these fluctuations are expected to be pronounced.

\section{Stochastic process}

\subsection{Stochastic variable}

A stochastic process is one involving random forces, and is described through a so-called stochastic variable (or random variable), which does not have a definite value, but is characterized instead by a probability distribution of values. 
Figure 5: Being squeezed by a water net, the protein chain rapidly collapses into the molten globule state, which slowly adjusts itself into the native state.

Practically everything we deal with in the macroscopic world involve random variables, from the position of a billiard ball to the value of a stock.

Einstein pointed out the essence of a stochastic variable in his theory of Brownian motion. He emphasized that every Brownian step we can observe is the result of a very large number of smaller random steps, which in turn are the result of a very number of even smaller steps, and so on, until we reach the cutoff imposed by atomic structure. This self-similarity leads to a Gaussian distribution, regardless of the underlying mechanism - a result known as the central limit theorem 9 .

\subsection{Brownian motion}

The simplest stochastic process is the Brownian motion of a single particle suspended in a medium. Its position $x(t)$ is a stochastic variable described by the Langevin equation

$$
m \ddot{x}=F(t)-\gamma \dot{x}
$$

Here, the force exerted by the medium on the particle is split into two parts: a randomly fluctuating force $F(t)$ and a friction $-\gamma \dot{x}$. The random force is a member of a statistical ensemble with the properties

$$
\begin{aligned}
\langle F(t)\rangle & =0 \\
\left\langle F(t) F\left(t^{\prime}\right)\right\rangle & =c_{0} \delta\left(t-t^{\prime}\right)
\end{aligned}
$$

where the brackets \langle\rangle denote ensemble average. The two forces are not independent, but related through the fluctuation-dissipation theorem:

$$
\frac{c_{0}}{2 \gamma}=k_{B} T
$$

where $k_{B}$ is Boltzmann's constant and $T$ is the absolute temperature, a property of the medium. 
The Langevin equation can be solved exactly, and also be simulated by random walk. Both methods lead to diffusion, in which the position has a Gaussian distribution with variance $\sqrt{2 D t}$, where $t$ is the time, and $D=c_{0} /\left(2 \gamma^{2}\right)$ is called the diffusion constant. An equivalent expression is Einstein's relation

$$
D=\frac{k_{B} T}{\gamma}
$$

Thus, a random force must generate energy dissipation, and the dissipation constant $\gamma$ can be deduced from the variance of the distribution of positions.

\subsection{Monte Carlo}

If a particle undergoes Brownian motion in the presence of a regular (nonrandom) external force $G(x)$, we may not be able to solve the Langevin equation exactly, but we can still simulate it on a computer by conditioned random walk, as follows. We first generate a random trial step, but accept it only according to the Monte Carlo algorithm. Let $E$ be the potential energy corresponding to the external force $G$. Let $\Delta E$ be the energy change in the proposed update. The algorithm is as follows:

- if $\Delta E \leq 0$, accept it;

- if $\Delta E>0$, accept it with probability $\exp \left(-\Delta E / k_{B} T\right)$.

The last condition simulates thermal fluctuations, which may drive the system to a higher energy. After a sufficiently large number of updates, the sequence of state generated will yield a canonical ensemble with temperature $T$. That is, the Monte Carlo procedure tends to minimize not the energy, but the free energy.

Mathematically speaking, conditioned random walk simulates a generalized Langevin equation, as indicated in the following:

$$
m \ddot{x}=\underset{\text { Treat via random walk }}{[F(t)-\gamma \dot{x}]}+\underset{\text { Treat via Monte Carlo }}{G(x) .}
$$

Of course, we could integrate the whole equation as a stochastic differential equation, as an alternative to Monte Carlo. The equivalence of these two methods is illustrated by example in the appendix of Ref.[15.

\section{CSAW}

In protein folding, we are dealing with the Brownian motion of a chain with interactions. All we need to do, in principle, is to generalize conditioned random walk to conditioned SAW (self-avoiding walk). The resulting model is called CSAW (conditioned self-avoiding walk).

We can generate a SAW representing an unfolded protein chain by the pivot algorithm [10] [11, as follows. Choose an initial chain in 3D continuous space, and hold one end of the chain fixed. 
- Choose an arbitrary point on the chain as pivot.

- Rotate the end portion of the chain rigidly about the pivot (by changing the torsional angles at the pivot point).

- If this does not result in any overlap, accept the configuration, otherwise repeat the procedure.

By this method, we can generate a uniform ergodic ensemble of SAW's, which simulates a Langevin equation of the form

$$
m_{k} \ddot{\mathbf{x}}_{k}=\mathbf{F}_{k}(t)-\gamma_{k} \dot{\mathbf{x}}+\mathbf{U}_{k}, \quad(k=1, \cdots, N)
$$

where the subscripts $k$ label the residues along the chain. The terms $\mathbf{U}_{k}$ denote the regular (non-random) forces that maintain the rigid bonds between successive residues, and that prohibit the residues from overlapping one another.

We now add other regular forces $G_{k}$, which include the hydrophobic interaction and hydrogen-bonding. Treating this force via Monte Carlo results in CSAW, which simulates a generalized Langevin equation as indicated in the following:

$$
m_{k} \ddot{\mathbf{x}}_{k}=\left(\mathbf{F}_{k}-\gamma_{\text {Treat via SAW }} \dot{\mathbf{x}}+\mathbf{U}_{k}\right)+\underset{\text { Treat via Monte-Carlo }}{\mathbf{G}_{k} .}(k=1 \cdots N)
$$

Now we shall specify the forces $G_{k}$ explicitly.

\section{Implementation of CSAW}

To reiterate, the system under consideration is a sequence of centers corresponding to $C_{\alpha}$ atoms, connected by planar "cranks". The degrees of freedom of the system are the pairs of torsional angles $\left\{\phi_{i}, \psi_{i}\right\}$ specifying the relative orientation of two successive cranks. There are $O$ and $H$ atoms attached to each crank, through rigid bonds lying in the same plane as the crank. The residues can differ from one another only through the side chains attach to $C_{\alpha}$, and there are 20 of them to choose from. As indicated in Fig.6, the center of the side chain is located at an apex of a tetrahedron with $C_{\alpha}$ at the center.

We can start with a chain of bare cranks, and then add other components one by one, as desired. We can first represent the side chains by hard spheres, and put in the atoms in a more elaborate version. In this manner, we can tinker with different degrees of buildup, and investigate the relative importance of each element.

For Monte Carlo, we take the energy $E$ to be

$$
\begin{aligned}
E & =-g_{1} K_{1}-g_{2} K_{2} \\
K_{1} & =\text { Total contact number of all hydrophobic residues } \\
K_{2} & =\text { Number of hydrogen bonds }
\end{aligned}
$$


Figure 6: The side chain is at the apex of a tetrahedron with $C_{\alpha}$ at the center.

The first term in $E$ expresses the hydrophobic effect. The contact number of a residue is the number of atoms touching its side chain. In the simplest version, in which we do not explicitly put in the side chain, the contact number is simply the number of atoms in contact with $C_{\alpha}$, not counting the other $C_{\alpha}$ 's lying next to it along the chain. This is illustrated in Fig.7a.

The contact number measures how well a residues is being shielded from the medium. When two hydrophobic residues are in contact, the total contact number increases by 2 , an this induces an effective attraction between hydrophobic residues. The unfolded chain corresponds to $g_{1}=0$.

The second term in $E$ describes hydrogen bonding. As illustrated in Fig.7b, a hydrogen bond is deemed to have formed between $O$ and $H$ from different cranks when

- the distance between $O$ and $H$ is $2.5 \mathrm{~A}$, within given tolerance;

- The bonds $C=O$ and $N-H$ are antiparallel, within given tolerance.

Only the combinations $g_{1} / k_{B} T$ and $g_{2} / k_{B} T$ appear in the Monte Carlo procedure. They are treated as adjustable parameters.

Note that $E$ only includes the potential energy. We can leave out the kinetic energy because it contributes only a constant factor to the configurational probability of the ensemble. 
Figure 7: (a) The shaded hydrophobic residue illustrated here has four contact neighbors. The permanent neighbors along the chain are not counted. (b) Hydrogen bonding occurs between $O$ and $H$ on the main chain, from different residues.

\section{$6 \quad$ Exploratory runs}

It is instructive to run the program with minimal components, as described in Refs. [7] [8]. For a chain of 30 residues, the main findings are the following:

- Under hydrophobic forces alone, without hydrogen-bonding, the chain folds into a reproducible shape. This shows that the hydrophobic effect alone can produce tertiary structure. There is no secondary structure in this case, and the chain rapidly collapses to the final structure without passing through an intermediate state..

- When there is no hydrophobic force and the interaction consists purely of hydrogen-bonding, the chain rapidly folds into one long alpha helix.

- When both hydrophobic force and hydrogen bonding are taken into account, secondary structure emerges. The folding process exhibits twostage behavior, with a fast collapse followed by slow "annealing", in qualitative agreement with experiments.

We now recount some simulations of realistic protein fragments.

\section{Folding pathways and energy landscape}

Chignolin is a synthetic peptide of 10 residues [13, in the shape of a "beta hairpin" - a turn in a beta sheet as depicted in Fig.3. Jinzhi Lei [14] of Tsinghua 
Figure 8: Folding of Chignolin, a beta hairpin with ten residues.

University modeled it in CSAW, with side chains modeled as hard spheres. The native state emerges after about 70000 trial steps, as shown in Fig.8. The computation took less than 5 minutes on a work station. In contrast, an MD simulation on the same work station did not reach the native state in one month's computation. The run was repeated 100 times independently, to obtain an ensemble of folding paths.

To display the folding pathways, we project them onto a two-dimensional subspace of the configuration space, chosen as follows. Define a $10 \times 10$ distance matrix $D_{i j}=\left|\mathbf{R}_{i}-\mathbf{R}_{j}\right|$, where $\mathbf{R}_{i}$ is the vector position of the $i$ th $C_{\alpha}$. Let its eignevalues be $\lambda_{1}, \ldots, \lambda_{10}$ in ascending order. Through experimentation, we find that it is best to project the pathways onto the $\lambda_{1}-\lambda_{10}$ plane, and we rotate the viewpoint to obtain the clearest representation. This is achieved by using $\lambda_{1}$ and $\lambda_{1}+\lambda_{10}$ as axes. Fig.9 shows the evolution of 100 folding paths. We can see that the ensemble of 100 points, identified by given shading, migrates towards an attractor as time goes on. The energy landscape is shown below the migration map.

In Fig.10 we show 4 individual paths. They get trapped in various local pockets, and breakout after long searches for outlets. In this respect, the paths are similar to Levy flights.

Finally, in Fig.11, we exhibit the elastic property of the protein chain by plotting the energy as a function of molecular radius, in a semilog plot. The behavior is consistent with an exponential force law. The flat portion in the middle corresponds to the breaking of hydrogen bonds that held the beta hairpin together. 
Figure 9: Evolution of 100 folding paths of Chignolin. The ensemble evolves towards an attractor. Lower panel shows the energy landscape. See text for explanation of the axes. 
Figure 10: Invidual pathways in the folding of Chignolin. Starting point are marked with an open circle, and endpoints are marked 1.

Figure 11: Elastic property of Chignolin: semilog plot of potential energy vs. radius, averaged over an ensemble of 50 samples. The flat part corresponding to the breaking of hydrogen bonds. The general shape of the curve is consistent with an exponential force law. Energy unit is not calibrated. 
Figure 12: Fractions of Polyalanine at various stages of folding, as functions of time. Picuture at top shows the native state of the protein fragment.

\section{Nucleation and growth of an alpha helix}

Next we report on Polyalanine $\left(\mathrm{Ala}_{20}\right)$, a protein fragment of 20 identical amino acids alanine, which is hydrophobic 15. The native state is known to be a single alpha helix. We tune $g_{1} / k_{B} T$ and $g_{2} / k_{B} T$ to maximize helical content.

An ensemble of 100 folding paths was generated. Fig.12 shows the fractions of unfolded, intermediate, and folded molecules, as functions of time. The solid curves are fits made according to a specific model, in which the molecular radius reaches equilibrium first, while the helical content continues to grow. The helical growth is described by a set of rate equations, while the relaxation of the radius is akin to that of an elastic solid. This shows that the tertiary structure was established before the secondary structure, and their evolutions are governed by different mechanisms.

Fig.13 shows a contour plot of the ensemble average of helicity, with time on the horizontal axis, and residue number along the vertical. We can see that the alpha helix grew from two specific nucleation points.

\section{All-atom model}

Finally we show some preliminary results of Weitao Sun [16] of Tsinghua University on the histone $1 \mathrm{~A} 7 \mathrm{~W}$, which has 68 residues. This is a test of an all-atom 
Figure 13: Contour map of ensemble average of helicity as a function of time and residue sequence, in the folding of Polyalanine. Nuclearion occured near the two positions marked by arrows.

CSAW model, in which atoms on the side chains are explicitly included. The model also includes the electrostatic interactions among all atoms. Fig.14 compares the simulated shape of the protein with the native state. It was found that inclusion of electrostatic interactions makes a noticeable improvement.

The main purpose of this calculation is to study the evolution of the dynamical structure function

$$
S(k, \omega)=\left\langle|n(\mathbf{k}, \omega)|^{2}\right\rangle
$$

where $n(\mathbf{k}, \omega)$ is the space-time Fourier transform of the particle density, and \langle\rangle denotes ensemble average. In principle, this function can be experimentally

Figure 14: Folding the histone 1A7W (68 residues) with an all-atom CSAW model including electrostatic interactions. 
Figure 15: 
measured via inelastic x-ray scattering. A peak occurring at particular $k, \omega$ will indicate the existence of an excitation mode. The integral of $S(k, \omega)$ over $k$ will yield the normal mode spectrum, and the integral over $\omega$ will yield the static structure factor. Preliminary results are shown in Fig.15. We see that at update $=3000$ the collective modes of the final structure have not yet formed, but they emerge at update $=5000$. The lower panel of Fig. 15 shows details of a sound-wave mode with constant velocity.

\section{Discussion and outlook}

In treating protein folding as a physical process, the CSAW model differs from MD in two important aspects, namely

- irrelevant degrees of freedom are ignored;

- the environment is treated as a stochastic medium.

These, together with simplifying treatment of interactions, enable the model to produce qualitatively correct results with minimal demands on computer time.

An important simplification is separating the hydrophobic effect and hydrogen bonding, as expressed by the separate terms in the potential energy (8). Since both effects arise physically from hydrogen bonding, it is not obvious that we can make such a separation. The implicit assumption is that hydrogen bonding with water involves only the side chains, while internal hydrogen bonding involves only atoms along the main chain. This property is supported by statistical data, but should be a result rather an assumption of the model. We should try to remedy this in an improved version of the model.

The successful examples discussed here deal either with the alpha helix or the beta hairpin. Our next goal is to study the formation of a beta sheet. This is a much more difficult problem, for it involves global instead of local properties of the protein chain. Not knowing which elements are crucial for the project, we have made the following enhancements to-date:

- All-atom side chains can now be installed, with fractional hydrophobicity.

- Electrostatic interactions among all atoms can be included.

- Hard-sphere repulsions between atoms can be replaced by Lennard-Jones potentials.

- Hydrogen bonds can switch among qualifying partners, with given probability.

We hope to make progress on this problem. 


\section{References}

[1] C. Branden and J. Tooze, Introduction to Protein Structure, 2nd ed., (Garland Publishing, New York, 1999).

[2] V. Daggett and A.R. Fersht, Nat. Rev.: Mol. Cell Biol. 4, 497 (2003).

[3] M. Kanehisa, Post-Genome Informatics, (Oxford University Press, Oxford, 2000).

[4] H.A. Scheraga, M. Khalili, and A. Liwo, Annu. Rev. Phys. Chem., 58, 57 (2007).

[5] K. Huang. Lectures on Statistical Physics and Protein Folding (World Scientific Publishing, Singapore, 2005).

[6] P. Flory, Principles of polymer chemistry (Cornell University Press, London, 1953).

[7] K. Huang, "CSAW: Dynamical model of protein folding", arXiv:cond-mat/0601244 v1 12 Jan 2006.

[8] K. Huang, Biophys. Rev. Lett., 2, 139 (2007).

[9] K. Huang. Introduction to Statistical Physics (Taylor \& Francis, London, 2001) Chaps.16,17.

[10] B. Li, N. Madras, and A.D. Sokal, J. Stat. Phys. 80, 661 (1995).

[11] T. Kennedy, J. Stat. Phys. 106, 407 (2002).

[12] M. Matsumoto, S. Saito, and I. Ohmine, Nature, 416, 409 (2002).

[13] A. Suenga et. al. Chem. Asian J. 2, 591 (2007).

[14] L.Z. Lei (unpublished).

[15] L.Z. Lei and K. Huang, "Dynamics of alpha-helix formation in the CSAW model", arXiv 07063256 v1 [cond-mat.soft] 22 Jun 2007.

[16] W.T. Sun (unpublished). 
This figure "Ala_nucleate.jpg" is available in "jpg" format from: http://arxiv.org/ps/0707.2388v1 
This figure "Ala_rates.jpg" is available in "jpg" format from: http://arxiv.org/ps/0707.2388v1 
This figure "AlphaBeta.jpg" is available in "jpg" format from: http://arxiv.org/ps/0707.2388v1 
This figure "ChainDetails.jpg" is available in "jpg" format from: http://arxiv.org/ps/0707.2388v1 
This figure "Elastic1.jpg" is available in "jpg" format from: http://arxiv.org/ps/0707.2388v1 
This figure "FoldingStages.jpg" is available in "jpg" format from: http://arxiv.org/ps/0707.2388v1 
This figure "Histone.jpg" is available in "jpg" format from: http://arxiv.org/ps/0707.2388v1 
This figure "Myoglobin.jpg" is available in "jpg" format from: http://arxiv.org/ps/0707.2388v1 
This figure "SchematicChain.jpg" is available in "jpg" format from: http://arxiv.org/ps/0707.2388v1 
This figure "Skw.jpg" is available in "jpg" format from: http://arxiv.org/ps/0707.2388v1 
This figure "chig_folding.jpg" is available in "jpg" format from: http://arxiv.org/ps/0707.2388v1 
This figure "chig_landscape.jpg" is available in "jpg" format from: http://arxiv.org/ps/0707.2388v1 
This figure "chig_paths.jpg" is available in "jpg" format from: http://arxiv.org/ps/0707.2388v1 
This figure "interactions.jpg" is available in "jpg" format from: http://arxiv.org/ps/0707.2388v1 
This figure "water.jpg" is available in "jpg" format from: http://arxiv.org/ps/0707.2388v1 